\title{
Der Erste Weltkrieg als deutsch-französischer Erinnerungsort?
}

Zwischen nationalem Gedenken und europäischer Geschichtspolitik

La Première guerre mondiale comme lieu de mémoire franco-allemand? Entre

commémoration nationale et politique de la mémoire européenne

The First World War as a Franco-German " realm of memory "? Between

national commemoration and a politics of European memory

Johannes Grossmann

\section{OpenEdition}

\section{Journals}

Édition électronique

URL : http://journals.openedition.org/ceg/2118

DOI : $10.4000 /$ ceg. 2118

ISSN : 2605-8359

Éditeur

Presses Universitaires de Provence

Édition imprimée

Date de publication : 15 juin 2014

Pagination : 207-220

ISSN : 0751-4239

\section{Référence électronique}

Johannes Grossmann, « Der Erste Weltkrieg als deutsch-französischer Erinnerungsort? », Cahiers d'Études Germaniques [Online], 66 | 2014, Online erschienen am: 17 Dezember 2017, abgerufen am 19 November 2020. URL : http://journals.openedition.org/ceg/2118; DOI : https://doi.org/10.4000/ceg. 2118 


\section{Der Erste Weltkrieg als deutsch- französischer Erinnerungsort? Zwischen nationalem Gedenken und europäischer Geschichtspolitik}

Johannes GROSSMANN

Université de Tübingen

\section{Konzepte}

\section{Erinnerungsort}

Ganz offensichtlich hatte Pierre Nora nicht vorausgeahnt, dass er durch die Herausgabe seines ursprünglich auf drei Bände angelegten Kompendiums französischer "lieux de mémoire" die geschichtswissenschaftliche Forschung nachhaltig beeinflussen würde. Denn erst gegen Ende seiner zwischen 1984 und 1992 erschienenen und letztlich auf sieben Bände angewachsenen Nationalgeschichte "zweiten Grades", welche die Nation und ihre Vergangenheit nicht als realhistorische Größe, sondern als ein auf Symbolen und Mythen basierendes Konstrukt verstand, präsentierte er den Lesern eine Definition seines zentralen Analysebegriffes: "Lieu de mémoire, donc: toute unité significative, d'ordre matériel ou idéel, dont la volonté des hommes ou le travail du temps a fait un élément symbolique du patrimoine mémoriel d'une quelconque communauté",2.

Eine weiterreichende Definition, die eine Übertragung von Noras Konzept auf andere nationale oder nicht-nationale Kontexte ermöglichte, entwickelten Étienne François und Hagen Schulze. In ihrer Adaption betonten sie vor allem die identitätsstiftende Funktion und den dynamischen Charakter von Erinnerungsorten :

\footnotetext{
${ }^{1}$ Pierre NORA, "Comment écrire l'histoire de la France?", in ders. (Hrsg.), Les lieux de mémoire, Bd. III: Les France, Teilbd. 1, Paris, Gallimard, 1992, S. 9-32, hier S. 25.

${ }^{2}$ Ibid. S. 20
} 
Es handelt sich um langlebige, Generationen überdauernde Kristallisationspunkte kollektiver Erinnerung und Identität, die in gesellschaftliche, kulturelle und politische Üblichkeiten eingebunden sind und die sich in dem Maße verändern, in dem sich die Weise ihrer Wahrnehmung, Aneignung, Anwendung und Übertragung verändert.

Die Dynamik der von ihnen untersuchten gedächtnisgeschichtlichen Prozesse und das aktive Moment im Handeln der daran beteiligten Akteure unterstrichen François und Schulze außerdem, indem sie sich bewusst dafür entschieden, "lieu de mémoire" nicht mit dem bis dahin im Deutschen gebräuchlichen Begriff "Gedächtnisort", sondern mit dem Begriff "Erinnerungsort" zu übersetzen ${ }^{4}$.

Definition und Begriffswahl von François und Schulze sollen für die nachfolgenden Überlegungen übernommen, gleichzeitig aber um eine scheinbar beiläufige, tatsächlich aber für die Analyse von Erinnerungsorten unverzichtbare Bemerkung Noras ergänzt werden. Demzufolge zeichnen drei Eigenschaften einen Überrest als "lieu de mémoire" aus ${ }^{5}$. Dazu zählt erstens seine materielle Qualität, wobei "materiell” nicht unbedingt gegenständlich bedeuten muss. So kann der Überrest beispielsweise ein konkreter geographischer Ort, ein Zeitpunkt, eine Person oder ein Text sein, aber auch ein fiktiver Ort oder eine Legende. Dem Überrest muss zweitens ein symbolischer Wert zugemessen sein. Er muss also in einem gruppenspezifischen Bezugsrahmen "für etwas stehen" und dabei drittens einen funktionalen Gehalt haben, also auf die jeweilige Gegenwart bezogen sein. Dieser funktionale Gehalt kann sich wandeln und dadurch auf den symbolischen Wert des Erinnerungsortes zurückwirken.

\section{Geteilte, parallele und gemeinsame Erinnerungsorte}

Noras Ansatz wurde rasch auf andere Länder übertragen. Bis 2010 entstanden Sammelbände und Monographien zu italienischen, deutschen, österreichischen, niederländischen, luxemburgischen, russischen, belgischen und schweizerischen Erinnerungsorten ${ }^{6}$. Gleichzeitig versuchten Historiker, Noras Fixierung auf die Nation zu dekonstruieren und die Existenz von regionalen, lokalen und milieubezogenen Gedächtnisorten, von

\footnotetext{
3 Étienne François, Hagen SchUlze, "Einleitung", in dies. (Hrsg.), Deutsche Erinnerungsorte, Bd. I, München, Beck, 2000, S. 9-24, hier S. 18.

4 Vgl. Tilmann RoBBE, Historische Forschung und Geschichtsvermittlung. Erinnerungsorte in der deutschsprachigen Geschichtswissenschaft, Göttingen, V\&R unipress, 2009 , S. 124-126.

5 Pierre NORA, "Entre mémoire et histoire. La problématique des lieux", in ders. (Hrsg.), Les lieux de mémoire, Bd. I, La République, Paris, Gallimard, 1984, S. XV-XLII, hier S. XXXIV.

${ }^{6}$ Vgl. den Überblick von Kornelia KoczaL, "Erinnerungsorte. Über die Karriere eines folgenreichen Konzepts", in Hans Henning HAHN, Robert TRABA (Hrsg.), Deutsch-Polnische Erinnerungsorte, Bd. 4, Reflexionen, Paderborn, Schöningh, 2013, S. 79-106.
} 
epocheneigenen, grenzüberschreitenden, binationalen, transnationalen und europäischen Erinnerungsorten nachzuweisen ${ }^{7}$. Gerade die deutschfranzösische Beziehungsgeschichte diente dabei wiederholt als Untersuchungsfeld für erinnerungsgeschichtliche Zusammenhänge und Narrative jenseits nationalgeschichtlicher Engführungen ${ }^{8}$.

Anknüpfend daran soll hier der Frage nachgegangen werden, ob und inwiefern es sich beim Ersten Weltkrieg um einen deutsch-französischen Erinnerungsort handelt. In Anlehnung an ein derzeit in der Abschlussphase befindliches Forschungsprojekt von Hans Henning Hahn und Robert Traba zur deutsch-polnischen Erinnerungsgeschichte ${ }^{9}$ und in systematisierender Bezugnahme auf die von Nora beschriebene Trias aus materiellem, symbolischem und funktionalem Gehalt lassen sich dabei drei verschiedene Typen bi- bzw. multinationaler Erinnerungsorte ausmachen :

Ein "geteilter Erinnerungsort" (im doppelten Sinne von partagé und divisé) ist demnach ein in materieller Hinsicht eindeutig bestimmbarer Überrest, dessen symbolischer und funktionaler Gehalt aber in Deutschland ein anderer ist als in Frankreich. "Parallele Erinnerungsorte" sind in materieller Hinsicht eigentlich zwei verschiedene Überreste, die aber im jeweiligen nationalen Gedächtnis einen ähnlichen bzw. vergleichbaren symbolischen und funktionalen Gehalt aufweisen. Als "gemeinsamer Erinnerungsort" bezeichnet werden kann schließlich ein in materieller Hinsicht eindeutig bestimmbarer Überrest, dessen symbolischer und funktionaler Gehalt in beiden nationalen Erinnerungskulturen und -kontexten (weitgehend) übereinstimmt.

\footnotetext{
${ }^{7}$ Vgl. zusammenfassend RoBBE, Historische Forschung, insbesondere S. 145-181. Siehe außerdem neuerdings: Pim den BoER, Heinz DuchHARDT, Georg KREIS, Wolfgang SchMALE (Hrsg.), Europäische Erinnerungsorte, 3 Bde., München, Oldenbourg, 2012.

${ }^{8}$ Siehe insbesondere Étienne FrançOIS, Hannes SIEGRIST, Jakob Vogel (Hrsg.), Nation und Emotion. Deutschland und Frankreich im Vergleich, 19. und 20. Jahrhundert, Göttingen, Vandenhoeck \& Ruprecht, 1995; Horst MöLLER, Jacques MORIZET (Hrsg.), Franzosen und Deutsche. Orte der gemeinsamen Geschichte, München, Beck, 1996; Rainer HUDEMANN (Hrsg.), Stätten grenzüberschreitender Erinnerung. Spuren der Vernetzung des Saar-Lor-LuxRaumes im 19. und 20. Jahrhundert - Lieux de la mémoire transfrontalière. Traces et réseaux dans l'espace Sarre-Lor-Lux aux XIX et XX siècles, Saarbrücken, 3. Aufl., 2009, http://www. memotransfront.uni-saarland.de; Thomas Keller (Hrsg.), Cahiers d'Études Germaniques, Nr. 53, 2/ 2007, Lieux de migration/ lieux de mémoire franco-allemands.

Hans Henning Hahn, Robert TRABA (Hrsg.), Deutsch-Polnische Erinnerungsorte, 5 Bde., Paderborn, Schöningh, 2012-2013. Zwar wird in diesem Projekt begrifflich zwischen den nachstehend genannten drei Typen von Erinnerungs- bzw. Gedächtnisorten unterschieden, die Differenzierung zwischen "gemeinsamen" und "geteilten" Gedächtnisorten bleibt jedoch unscharf und wird nicht theoretisch begründet.
} 


\begin{tabular}{|c|c|c|c|}
\hline & 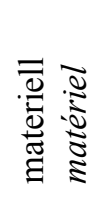 & 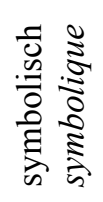 & 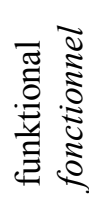 \\
\hline $\begin{array}{l}\text { geteilter Erinnerungsort } \\
\text { lieu de mémoire partagé / divisé }\end{array}$ & $=$ & $\neq$ & $\neq$ \\
\hline $\begin{array}{l}\text { paralleler Erinnerungsort } \\
\text { lieu de mémoire parallèle }\end{array}$ & $\neq$ & $=$ & $=$ \\
\hline $\begin{array}{l}\text { gemeinsamer Erinnerungsort } \\
\text { lieu de mémoire commun }\end{array}$ & $=$ & $=$ & $=$ \\
\hline
\end{tabular}

\title{
Geschichtspolitik
}

Mit dem Terminus der "Geschichtspolitik" enthält der Titel dieses Beitrags einen weiteren Begriff, der einer genaueren Bestimmung bedarf. Edgar Wolfrum definiert Geschichtspolitik als

\begin{abstract}
ein Handlungs- und Politikfeld, auf dem verschiedene politische Akteure die Vergangenheit mit bestimmten Interessen befrachten und in der Öffentlichkeit um Zustimmung ringen. Dabei bestehen vielfältige Interdependenzen zwischen Politik, Publizistik, Wissenschaft und öffentlicher Meinung. Moderne Demokratien werden durch Deutungszusammenhänge mobilisiert; Geschichte kann hier ein wichtiges Vehikel sein, um Zusammenhänge zwischen diffusen Gruppen zu schaffen, Auseinandersetzungen zu polarisieren, Skandale zu provozieren oder den politischen Gegner zu delegitimieren. ${ }^{10}$
\end{abstract}

Dieser Definition zufolge sind Erinnerungsorte untrennbarer Bestandteil des geschichtspolitischen Feldes. Denn ihr symbolischer und ihr funktionaler Gehalt sind das Resultat eines ständigen Aushandlungsprozesses zwischen verschiedenen Sphären der Geschichtskultur. Unterscheiden lassen sich dabei idealtypisch die von individuellen Akteuren getragene, von persönlicher und

${ }^{10}$ Edgar WOLFrUM, "Geschichtspolitik in der Bundesrepublik Deutschland 1949-1989”, in Aus Politik und Zeitgeschichte, Bd. 45, 1998, S. 3-15, hier S. 5. 
familiärer Erinnerung geprägte "private Geschichtskultur", die von (nichtstaatlichen) gemeinschaftlichen Akteuren dominierte, beispielsweise von (privaten) Museen, Geschichtsvereinen oder geschichtswissenschaftlicher Forschung vertretene "ungelenkte institutionelle Geschichtskultur" sowie die staatliche Domäne der "gelenkten institutionellen Geschichtskultur", wie sie beispielsweise in offiziellen Verlautbarungen, der Errichtung von Denk- und Mahnmälern oder den Inhalten von Lehrplänen und Schulbüchern zum Ausdruck kommt ${ }^{11}$.

Letzten Endes muss sogar das Erinnerungsort-Konzept selbst als ein Produkt beziehungsweise Instrument von Geschichtspolitik angesehen werden - sei es im Dienste nationaler, regionaler und lokaler Identitätsstiftung oder im Dienste von Annäherung, Integration und Völkerverständigung. So verweist Tilmann Robbe zu Recht darauf, dass Noras Begriff des "lieu de mémoire" rasch vom Staat vereinnahmt und dadurch

zum Werkzeug dessen geworden [sei], das zu beschreiben er konzipiert worden war. Das Polaritätsverhältnis zwischen wissenschaftlicher Deskription und Reflexion einerseits und der Nutzung als Instrument der Selbstvergewisserung andererseits, das in der Entwicklung des französischen Projektes sichtbar wird, zieht sich durch alle nachfolgenden Verwendungen des Begriffs der Erinnerungsorte. ${ }^{12}$

Als "Leitbegriff staatlicher wie gesellschaftlicher Vergangenheitspflege ${ }^{13}$ " sind Gedächtnis- bzw. Erinnerungsorte dadurch selbst zur geschichtspolitischen Verfügungsmasse geworden.

\section{Konjunkturen}

\section{4/18 bis 1961/62 - Von der Kriegsschuldfrage zur einmütigen Amnesie}

Im Hinblick auf die "private Geschichtskultur" und das individuelle Gedenken in der Zeit unmittelbar nach dem Ersten Weltkrieg lässt sich in beiden Ländern eine tiefe Kluft zwischen der Erinnerung der Frontsoldaten und der Erinnerung der übrigen Bevölkerung beobachten. So beruhte das Bild, das sich die breite Bevölkerung von der Front machte, auf einer mangelnden Kenntnis der Kriegswirklichkeit und der "zu guten Teilen auf

\footnotetext{
11 Vgl. Tobias ARAND, "Geschichtskultur des Ersten Weltkriegs - Prolegomena zu einer Systematik”, in ders. (Hrsg.), Die „Urkatastrophe“ als Erinnerung. Geschichtskultur des Ersten Weltkriegs, Münster, Zentrum für Lehrerbildung, 2006, S. 1-14, hier S. 8-10.

12 ROBBE, Historische Forschung, S. 111.

13 Ibid.
} 
reinem Wunschdenken basierende[n] Propaganda ${ }^{14 \%}$. In Deutschland konnte sich daher kein allseits akzeptiertes Erinnerungsnarrativ entwickeln. Veteranenverbände radikalisierten sich, stellten die neue politische Ordnung in Frage und führten den "Krieg im Frieden" ${ }^{15}$ " weiter gegen "Erfüllungspolitiker", "Kriegsgewinnler", "Bolschewisten", "Juden" ... Auch in Frankreich war die Erinnerung der Soldaten alles andere als deckungsgleich mit den Erfahrungen der an der "Heimatfront". Beide Gruppen konnten sich jedoch im Narrativ der aufopferungsvollen und letztlich erfolgreichen Verteidigung der Nation wiederfinden. Die meisten Veteranenverbände huldigten einem - bei aller Kritik am parlamentarischen System - republiktreuen Antimilitarismus und Pazifismus, der auch die politischen Entscheidungsprozesse nachhaltig beeinflusste ${ }^{16}$.

In der "ungelenkten" und "gelenkten institutionellen Geschichtskultur" in dieser Phase kaum voneinander zu trennen - blieb die Kriegsschuldfrage das dominierende Thema. Historiker erarbeiteten im Auftrag der Regierungen "Farbbücher", um den eigenen Anteil am Ausbruch des Krieges zu bagatellisieren und die Verantwortung der Gegenseite zuzuschieben ${ }^{17}$. Was interessierte, war nicht der Krieg selbst, sondern seine Vorgeschichte: "Weder in Deutschland noch in Frankreich [...]", so Gerhard Hirschfeld, "fand der Erste Weltkrieg in den 1920er und 1930er Jahren eine historiographische Darstellung, die jenseits eng gefasster militärgeschichtlicher Fragestellungen wissenschaftlichen Ansprüchen genügt hätte ${ }^{18,}$.

Die Erinnerungen der Frontsoldaten gingen erst unter dem Nationalsozialismus in den offiziellen staatlichen Diskurs über den Weltkrieg ein. Doch waren es nicht die nachdenklichen Töne, sondern die "mythische[n] Beschwörungen" vermeintlich heroischer Episoden, denen die Nationalsozialisten Gehör verschafften ${ }^{19}$. Diese Erinnerung an den Ersten Weltkrieg war hochgradig selektiv und stand im Dienste der Machtsicherung und der "Wehrhaftmachung" - weshalb das Thema nach 1945 anrüchig erschien und die Erforschung des Krieges "in den ersten Jahrzehnten nach dem Zweiten Weltkrieg in Deutschland nahezu obsolet war ${ }^{20,}$. In Frankreich wiederum verlor die Erinnerung an den Ersten Weltkrieg durch die

\footnotetext{
${ }^{14}$ Gerd KRUMEICH, "Konjunkturen der Weltkriegserinnerung", in Rainer RothER (Hrsg.), Der Erste Weltkrieg 1914-1918. Ereignis und Wirkung, Berlin, Minerva, 2004, S. 68-74, hier S. 69.

5 Bernd Ulrich, Benjamin Ziemann (Hrsg.), Krieg im Frieden. Die umkämpfte Erinnerung an den Ersten Weltkrieg, Quellen und Dokumente, Frankfurt a. M., Fischer, 1997.

${ }^{16} \mathrm{Vgl}$. Antoine Prost, Les anciens combattants et la société française, 1914-1939, Bd. 3: Mentalités et idéologies, Paris, Presses de la Fondation Nationale de Sciences Politiques, 1977.

17 Sascha ZALA, Geschichte unter der Schere politischer Zensur. Amtliche Aktensammlungen im internationalen Vergleich, München, Oldenbourg, 2001, insbesondere S. 47-91.

18 Gerhard HiRsChFeld, "Der Erste Weltkrieg in der deutschen und internationalen Geschichtsschreibung", in Aus Politik und Zeitgeschichte, B 29-30, 2004, S. 3-12, hier S. 4.

${ }^{19}$ Ibid., S. 5.

${ }^{20}$ KRUMEICH, "Konjunkturen”, S. 71f.
} 
Niederlage von 1940 und den Wandel des Weltkriegshelden Philippe Pétain zum Erfüllungsgehilfen der deutschen Besatzungsmacht ihre Funktion als Kitt politischer, sozialer und ideologischer Gegensätze. Auch hier trat der Erste Weltkrieg als geschichtspolitischer Referenzpunkt und als Thema der Forschung nach 1945 vorerst in den Hintergrund ${ }^{21}$. Diese durch den Nationalsozialismus und die Vichy-Erfahrung ausgelöste, zumindest partielle, Amnesie kam auch darin zum Ausdruck, dass die Gespräche der deutschfranzösischen Schulbuchkommission 1951 in einer erstaunlich einmütigen Vereinbarung mündeten: "Die Dokumente erlauben es nicht, im Jahre 1914 irgendeiner Regierung oder einem Volk den bewussten Willen zu einem europäischen Kriege zuzuschreiben ${ }^{22}$."

\section{1/62 bis 1989/91 - Von der Fischer-Kontroverse zur Alltagsgeschichte}

Um diesen stillschweigenden Konsens war es 1961 geschehen, als der Hamburger Historiker Fritz Fischer die Kriegsschulddebatte mit seiner These vom deutschen Griff nach der Weltmacht ${ }^{23}$ neu belebte und damit nicht nur die deutsche Geschichtswissenschaft ${ }^{24}$, sondern auch die Weltkriegsforschung in anderen Ländern in Bewegung brachte. So setzte, befeuert durch die Auflösung des französischen Kolonialreiches, nun auch in Frankreich eine Diskussion über Hegemonialpolitik ein. Da gesellschaftliche und politische Widerstände eine quellengestützte historiographische Auseinandersetzung mit der jüngsten Vergangenheit behinderten, konzentrierte sich diese Debatte notgedrungen erst einmal auf die Entwicklungen des 19. und des frühen 20. Jahrhunderts ${ }^{25}$.

Im Mittelpunkt der nun auch in den Massenmedien ausgetragenen Debatten über die Verortung des Ersten Weltkriegs in seinem breiteren zeitlichen Umfeld stand jedoch nicht mehr nur die Kriegsschuldfrage. Vielmehr galt das Interesse nun vorrangig den wirtschaftlichen und sozialen Implikationen des Krieges, die zunehmend in vergleichender Perspektive

\footnotetext{
${ }^{21}$ Vgl. zu dieser Einschätzung auch den historiographiegeschichtlichen Überblick von Antoine Prost, Jay Winter, Penser la Grande Guerre. Un essai d'historiographie, Paris, Seuil, 2004, insbesondere S. 15-50.

${ }^{22}$ Zitiert nach: Romain FAURE, "Vom internationalen zum historiographischen Konflikt. Der Erste Weltkrieg in der Deutsch-Französischen Schulbuchkommission”, in Eckert. Das Bulletin, Nr. 11, 2012, S. 11-14, hier S. 12f.

${ }^{23}$ Fritz FISCHER, Griff nach der Weltmacht. Die Kriegszielpolitik des kaiserlichen Deutschland 1914/1918, Düsseldorf, Droste, 1961.

${ }^{24}$ Zum Verlauf der Debatte siehe zusammenfassend: Klaus Grosse Kracht, "Die Fischer-Kontroverse. Von der Fachdebatte zum Publikumsstreit", in ders., Die zankende Zunft. Historische Kontroversen in Deutschland nach 1945, Göttingen, Vandenhoeck \& Ruprecht, 2. Aufl., 2011, S. 47-67.

${ }_{25}$ Siehe dazu emblematisch Henri Brunschwig, Mythes et réalités de l'impérialisme colonial français, 1871-1914, Paris, Colin, 1960.
} 
untersucht wurden ${ }^{26}$. Dass diese - für die Entwicklung der Geschichtswissenschaft insgesamt prägende - Hinwendung zur Struktur- und Gesellschaftsgeschichte in den sechziger und siebziger Jahren alles andere als unpolitisch war, verdeutlichen die Auseinandersetzungen über die eng mit der Weltkriegshistoriographie verbundene These vom deutschen "Sonderweg ${ }^{27}$ ". Allerdings verliefen die Konfliktlinien nunmehr weniger entlang nationaler Grenzen, sondern entlang politischer Einstellungen und ideologischer Überzeugungen.

Eine gewisse Entpolitisierung der Diskussion ließ sich erst seit den späten siebziger Jahren beobachten, als alltags- und mentalitätsgeschichtliche Deutungsmuster einen anderen Blick auf den Ersten Weltkrieg ermöglichten. Das individuelle Gedenken der noch lebenden ehemaligen Frontsoldaten erhielt nun einen neuen Stellenwert. Sowohl in Frankreich als auch in der Bundesrepublik wurde der Kampf zwischen Mensch und Maschine vor der grauen Kulisse kraterübersäter Mondlandschaften zur vorherrschenden Meistererzählung vom Ersten Weltkrieg - illustriert durch Fotografien von zerschundenen Körpern, zerschossenen Bäumen und endlosen Schlachtfeldern. Kriegstagebücher, Feldpostkarten und Zeitzeugengespräche, von der Forschung als neue Quellen entdeckt, zeugten von der Inhumanität des industriellen Krieges und vom Zynismus der Armeeführung gegenüber den "einfachen" Soldaten ${ }^{28}$.

\section{9/91 bis heute - Auf dem Weg in eine gesamteuropäische Gedenkkultur?}

Die dritte und bislang letzte Phase der Erinnerung begann um 1989/91 und dauert bis heute an. Sie ist gekennzeichnet durch den Tod der letzten verbliebenen Zeitzeugen und damit durch jenen Übergang vom "kommunikativen" zum "kulturellen Gedächtnis" - man könnte auch sagen: von der Zeitgeschichte zur Geschichte -, in dessen Zuge Jan Assmann zufolge die vormals alltagsnahe, sprachgebundene, "lebendige" Erinnerung "auskristallisiert in die Formen der objektivierten Kultur", ihren "Gruppenund Gegenwartsbezug" verliert und zur identitätsstiftenden "Erinnerungsfigur" wird ${ }^{29}$.

Tatsächlich lässt sich seit etwa 25 Jahren beobachten, wie die alltags- und mentalitätsgeschichtliche Perspektive - zumindest in den westlichen Gesellschaften - von fast allen geschichts- und erinnerungspolitischen Akteuren internalisiert wird und die alten nationalen Meistererzählungen in

${ }^{26}$ HIRSCHFELD, "Der Erste Weltkrieg", S. 7.

27 Zur "Sonderwegs"-Debatte siehe zusammenfassend: Hans-Peter UllmanN, Politik im Deutschen Kaiserreich, 1871-1918, München, Oldenbourg, 2. Aufl., 2005, S. 53-62.

${ }^{28}$ HiRSCHFELD, "Der Erste Weltkrieg”, S. 8-10.

29 Jan AsSmanN, "Kollektives Gedächtnis und kulturelle Identität", in ders., Tonio HÖLSCHER (Hrsg.), Kultur und Gedächtnis, Frankfurt a. M., Suhrkamp, 1988, S. 9-19, hier S. $11 f$. 
Schulbüchern und Museen, in Zeitung, Film und Fernsehen ersetzt. Eine vergleichende beziehungsweise europäische Darstellung des Krieges, wie im 1992 eröffneten Historial de la Grande Guerre in Péronne ${ }^{30}$, im 2005 in europäischer Koproduktion gedrehten Kinohit Merry Christmas / Joyeux Noël über den "Weihnachtsfrieden" von $1914^{31}$ oder im 2008 erschienenen zweiten Band des deutsch-französischen Geschichtsbuchs ${ }^{32}$, ist nicht nur weithin akzeptiert, sondern vielmehr geschichtspolitisches Gebot.

Diese Entwicklung kollidiert jedoch mit einer Re-Politisierung und ReNationalisierung der Erinnerung in Ost- und Ostmitteleuropa seit dem Fall des Eisernen Vorhangs. Denn seit den neunziger Jahren wird die Geschichte der "vergessenen Front ${ }^{33 \text { " }}$ im Osten in den vormals kommunistisch regierten Ländern auf die geschichtspolitische Agenda gesetzt. Nicht selten wird dabei die Erinnerung an den Ersten Weltkrieg instrumentalisiert, um wiedererwachten ethnischen, nationalen und hegemonialen Forderungen Nachdruck zu verleihen. Der in siebzig Jahren ausgehandelte westeuropäische Erinnerungskonsens sieht sich dadurch erneut in Frage gestellt. Parallel dazu lässt sich eine Re-Individualisierung und Diversifizierung der Erinnerung im Internet und in neuen sozialen Medien beobachten ${ }^{34}$, die einen Resonanzraum für nationale Animositäten, Mystifizierung und historische Halbwahrheiten bildet und sich der Einbindung in einen europäischen Erinnerungskonsens entzieht.

\section{Verortungen}

\section{"1914” - Europa zwischen Selbstzerstörung und Selbstfindung}

Ist der Erste Weltkrieg nun ein deutsch-französischer Erinnerungsort? Oder vorsichtiger formuliert: Hat der Erste Weltkrieg deutsch-französische Erinnerungsorte hervorgebracht? Drei Beispiele sollen herausgegriffen werden, um nach Antworten auf diese Frage zu suchen und die Vielschichtigkeit der deutsch-französischen "Erinnerungslandschaft" im

\footnotetext{
${ }^{30}$ Siehe Sophie WAHNICH, Antoine TISSERON, “'Disposer des corps' ou mettre la guerre au musée. L'historial de Péronne, un musée d'histoire européenne de la guerre de 1914-1918”, in Tumultes, Nr. 16, 2001, S. 55-81.

31 Siehe Sylvia PALETSCHEK, "Der Weihnachtsfrieden 1914 und der Erste Weltkrieg als neuer (west-) europäischer Erinnerungsort. Epilog", in dies., Barbara KORTE, Wolfgang HOCHBRUCK (Hrsg.), Der Erste Weltkrieg in der populären Erinnerungskultur, Klartext, Essen, 2008, S. 213-220.

${ }_{32}$ Daniel Henri, Guillaume Le Quintrec, Peter Geiss (Hrsg.), Histoire/ Geschichte. Deutsch-französisches Geschichtsbuch, Gymnasiale Oberstufe, Bd. 2, Europa und die Welt vom Wiener Kongress bis 1945, Stuttgart, Klett, 2008, insbesondere S. 187-231.

${ }_{33}$ Gerhard P. Gross (Hrsg.), Die vergessene Front - der Osten 1914/15. Ereignis, Wirkung, Nachwirkung, Paderborn, Schöningh, 2006.

34 Gundula BAVEndAmM, "Der Erste Weltkrieg im Internet", in GRoss (Hrsg.), Die vergessene Front, S. 373-391.
} 
Hinblick auf den Ersten Weltkrieg zu verdeutlichen. Da wäre zunächst "1914" als das Jahr des Kriegsbeginns. Im deutsch-französischen Zusammenhang könnte "1914" zunächst einmal "Sarajevo 1914" bedeuten und damit auf einen geteilten Erinnerungsort verweisen. Geteilt, weil es sich einerseits um den gleichen materiellen Überrest handelt: das tödliche Attentat auf den österreichischen Thronfolger, Auslöser der "Julikrise" und Anlass des Kriegsausbruchs. Geteilt, weil sich dessen symbolischer und funktionaler Gehalt in Deutschland und Frankreich lange voneinander unterschieden. So stand "Sarajevo 1914" in Deutschland symbolisch für die serbische Aggression gegen den Bündnispartner und wurde funktionalisiert, um die "Nibelungentreue" gegenüber dem Habsburgerreich zu beschwören bzw. die Verantwortung für den Kriegsausbruch von sich zu weisen. In Frankreich galt "Sarajevo 1914" als Symbol für den Anachronismus des "Völkerkerkers" Österreich-Ungarn und den hegemonialen Drang des Deutschen Kaiserreiches und konnte somit ebenfalls zur Rechtfertigung des eigenen Verhaltens in der "Julikrise" funktionalisiert werden.

Später entwickelte sich der geteilte zu einem gemeinsamen Erinnerungsort: "Sarajevo 1914" stand nun für das unheilvolle Aufeinanderprallen nationaler und hegemonialer Interessen, im weiteren Sinne für die Selbstzerstörung und den "Untergang des alten Europa ${ }^{35, "}$. Diese Deutung entfaltete in den 1990er Jahren vor dem Hintergrund des jugoslawischen Bürgerkriegs ihre volle funktionale Wirkung. "Sarajevo 1914" wurde zum Warnzeichen für eine durch ethnische Konflikte drohende Rückkehr des Krieges nach Europa. Dass durch diesen erinnerungsgeschichtlichen Rekurs auch alte nationale Ressentiments zu neuem Leben erwachten, illustriert die damals gerade in Frankreich kursierende Furcht vor einem, durch die USA gestützten, neuen deutschen Hegemonialstreben in (Ost-)Europa ${ }^{36}$.

"1914" könnte im deutsch-französischen Zusammenhang aber auch für die "Union sacrée" und den "Burgfrieden" stehen, und damit auf einen parallelen Erinnerungsort verweisen. Parallel, weil es sich bei der in Frankreich von Staatspräsident Raymond Poincaré am 4. August 1914 verkündeten, wenig später durch den Regierungseintritt sozialistischer Politiker besiegelten "Union sacrée" im Zeichen der militärischen Bedrohung unzweifelhaft um ein anderes historisches Ereignis handelte als um den am gleichen Tag von Kaiser Wilhelm I. für das Deutsche Reich proklamierten "Burgfrieden", also die Zurückstellung aller ideologischen, sozialen und konfessionellen Konflikte für die Dauer des Krieges. Parallel, weil der symbolische und funktionale Gehalt dieser zwei Erinnerungsorte in den jeweiligen nationalen Kontexten vergleichbar waren. Sowohl die Verkündung der "Union sacrée" als auch die des "Burgfriedens" waren von vornherein als symbolische Akte

\footnotetext{
35 Volker R. Berghahn, Sarajewo, 28. Juni 1914. Der Untergang des alten Europa, München, DTV, 1997.

36 Vgl. beispielhaft für derartige Schreckensszenarien: Pierre-Marie Gallois (Hrsg.), Guerres dans les Balkans. La nouvelle Europe germano-américaine, Paris, Ellipses, 2002.
} 
konzipiert. Die Erinnerung daran wurde - weit über den Krieg hinaus - für die Wahrung der nationalen Einheit, die Disqualifizierung politischer Gegner und die Ruhigstellung revolutionärer Kräfte funktionalisiert ${ }^{37}$. Freilich entwickelte sich auch eine pazifistische bzw. "antiimperialistische" Gegenerinnerung, die "Union sacrée" und "Burgfrieden" als Symbole für das Versagen der Sozialisten in der "Julikrise" deutete. Heutzutage hat sich die Erinnerung an die "Union sacrée" und den "Burgfrieden" allerdings weitgehend von ihren nationalistischen und revolutionären Traditionslinien gelöst und ist, wie das Attentat von Sarajevo, die "Julikrise" und das "Augusterlebnis", in einer gemeinsamen deutsch-französischen bzw. westeuropäischen Erinnerung an die "Urkatastrophe" des Jahres 1914 aufgegangen.

\section{“Verdun” - Der Erste Weltkrieg als deutsch-französischer Krieg}

"Verdun", das wie kaum ein anderer Begriff für die deutsch-französische Dimension des Ersten Weltkriegs steht, beginnt seine gedächtnisgeschichtliche Karriere ebenfalls als geteilter Erinnerungsort. Der materielle Bezugspunkt in Deutschland und Frankreich ist der selbe: die Schlacht um Verdun, von Februar bis Dezember 1916, mehr als 300000 Tote, unzählige Verwundete, über 50 Millionen Granateinschläge, ein Landstrich von $260 \mathrm{~km}^{2}$ von Gräben durchzogen, von Kratern übersät, auf Dauer verödet. "Verdun" war zweifellos ein "Mikrokosmos des Ersten Weltkrieges" ${ }^{\prime 8}$. Doch im Gegensatz zu anderen Kriegsschauplätzen der Westfront wie an der Marne, an der Somme oder in Flandern, standen sich hier fast ausschließlich französische und deutsche Soldaten gegenüber. Der symbolische und funktionale Wert, der dieser Schlacht in Frankreich und Deutschland beigemessen wurde, unterschied sich zunächst deutlich. In Frankreich wurde "Verdun" zum Symbol für die erfolgreiche Verteidigung der Heimat unter großen Opfern ${ }^{39}$. In funktionaler Hinsicht diente "Verdun" der Beschwörung der nationalen Einheit und zur Rechtfertigung einer vorrangig defensiven, zunehmend rückständigen Militärstrategie. Über den zum "Helden von Verdun" stilisierten Oberkommandierenden Philippe Pétain wurde "Verdun" schließlich zum Referenzmythos des Vichy-Regimes ${ }^{40}$. In

\footnotetext{
${ }^{37}$ Siehe in vergleichender Perspektive: Wolfram Pyta, Carsten KRETSChMAnN (Hrsg.), Burgfrieden und Union sacrée. Literarische Deutungen und politische Ordnungsvorstellungen in Deutschland und Frankreich 1914-1933, München, Oldenbourg, 2011.

${ }^{38}$ Gerd KRUMEICH, "Verdun. Ein Ort gemeinsamer Erinnerung?", in MÖLlER, MORIZET (Hrsg.), Franzosen und Deutsche, S. 162-184, hier S. 167.

Zum Werdegang "Verduns" als französischer Gedächtnisort vgl. Antoine PROST, "Verdun", in NORA (Hrsg.), Les lieux de mémoire, Bd. II: La Nation, Teilbd. 3, Paris, Gallimard, 1986, S. 111-141; Serge BARCELlini, "Mémoire et mémoires de Verdun 1916-1996", in Guerres mondiales et conflits contemporains, Nr. 182, 1996, S. 77-98. 1992.

${ }^{40}$ Pierre Servent, Le mythe Pétain. Verdun ou les tranchées de la mémoire, Paris, Payot,
} 
Deutschland stand "Verdun" für die Tapferkeit des einsamen, von der Heimat im Stich gelassenen Frontsoldaten und wurde damit zum Referenzpunkt für die "Dolchstoßlegende".

Der geteilte Erinnerungsort "Verdun" wurde schließlich durch geschichtspolitische Interventionen zum gemeinsamen Erinnerungsort stilisiert. Die Nationalsozialisten sahen "Verdun" vor allem als Symbol für die vermeintliche deutsch-französische "Erbfeindschaft", die freilich als ehrenwerter Kampf der "besten Soldatenvölker der Alten Welt ${ }^{41}$ " gezeichnet wurde. Ganz auf dieser Linie lag das Treffen französischer und deutscher "Verdun-Kämpfer" in der Nacht vom 12. auf den 13. Juli 1936 vor dem monumentalen Beinhaus von Douaumont. Der dort geleistete Friedensschwur ließ sich - nur kurze Zeit nach der widerrechtlichen Remilitarisierung des Rheinlandes - von der nationalsozialistischen Propaganda als Demonstration der angeblichen deutschen Versöhnungsbereitschaft funktionalisieren. Der Mythos von Verdun wurde damit zum Instrument der von Otto Abetz und seiner Deutsch-französischen Gesellschaft im Auftrag der Dienststelle Ribbentrop betriebenen Annäherungspolitik, die zahlreiche französische Entscheidungsträger in ihrer besänftigenden Haltung gegenüber Deutschland bestärkt haben dürfte ${ }^{42}$.

Unbewusst knüpften François Mitterrand und Helmut Kohl an die in den dreißiger Jahren vollzogene Umdeutung an, als sie im September 1984 an gleicher Stelle mit ihrem Handschlag ein neues Kapitel in der Geschichte des Erinnerungsortes "Verdun" eröffneten. Auch Mitterrand und Kohl interpretierten "Verdun" als einen gemeinsamen Erinnerungsort, der symbolisch für den deutsch-französischen "Bruderkampf" stand. Sie betten diesen Erinnerungsort allerdings in einen anderen funktionalen Zusammenhang ein und instrumentalisierten ihn für die öffentliche Bestärkung der von Konrad Adenauer und Charles de Gaulle entworfenen Meistererzählung von der deutsch-französischen "Aussöhnung". Sie stellten "Verdun" damit in den Dienst einer verstärkten deutsch-französischen Annäherung und einer forcierten europäischen Integration ${ }^{43}$. Mit der Einrichtung eines internationalen Begegnungszentrums, des Centre Mondial de la Paix, im Bischofspalast von Verdun hat der Prozess der Umdeutung zu einem gemeinsamen Erinnerungsort 1990 seinen vorläufigen Abschluss gefunden.

\footnotetext{
${ }^{41}$ Wilhelm ZIEGLER, Verdun, Hamburg, Hanseatische Verlags-Anstalt, 1936, S. 188.

${ }^{42}$ Vgl. Roland RAY, Annäherung an Frankreich im Dienste Hitlers? Otto Abetz und die deutsche Frankreichpolitik 1930-1942, München, Oldenbourg, 2000, hier S. 147.

43 Vgl. Ulrich PfEIL, "Der Händedruck von Verdun. Pathosformel der deutschfranzösischen Versöhnung”, in Gerhard PAUL (Hrsg.), Das Jahrhundert der Bilder, Bd. 2: 1949 bis heute, Göttingen,Vandenhoeck \& Ruprecht, 2008, S. 498-505.
} 


\section{"Versailles" - Vom verlorenen Frieden zur deutsch-französischen "Aussöhnung”?}

Der letzte hier analysierte Erinnerungsort bezieht sich auf ein Ereignis, das zeitlich schon außerhalb der eigentlichen Kriegshandlungen liegt. "Versailles" 44 " verwies bereits vor dem Ersten Weltkrieg auf zwei parallele Erinnerungsorte, die als Symbole für die nationalstaatliche Einheit standen und als funktionale Referenzpunkte für die historische Legitimation der französischen Dritten Republik und des Deutschen Kaiserreichs dienten. In materieller Hinsicht handelte es sich allerdings nicht um ein und denselben Erinnerungsort, sondern um zwei verschiedene. Im französischen Fall wurde auf "Versailles" als historisches Machtzentrum Frankreichs Bezug genommen. Versinnbildlicht wurde dieser Bezug in der Nutzung des Schlosses als historisches Museum sowie in der an diesem Ort vollzogenen Wahl des französischen Staatspräsidenten. In Deutschland gedachte man der Kaiserproklamation vom 18. Januar 1871 im Spiegelsaal des Versailler Schlosses.

Nach dem Ersten Weltkrieg wurde "Versailles" zu einem geteilten deutsch-französischen Erinnerungsort. Materiell handelte es sich bei diesem "Versailles" ohne Zweifel um den Ort des Friedensvertrages von 1919. Die symbolischen und funktionalen Implikationen dieses Erinnerungsortes waren jedoch grundverschieden. In Frankreich galt "Versailles" - gerade in Anspielung auf das Jahr 1871 - nun als Symbol für die Wiedergewinnung der nationalen Ehre und der europäischen Hegemonialstellung. In funktionaler Hinsicht untermauerte "Versailles" den Anspruch Frankreichs, als Gestalter einer neuen internationalen und sozialen Friedensordnung aufzutreten und ein deutsches Widererstarken zu verhindern. Es verwundert kaum, dass der Erinnerungsort "Versailles" für die Franzosen in dem Maße an Bedeutung verlor, wie sich dieser Anspruch als Illusion erweisen sollte. In Deutschland wurde das "Schanddiktat von Versailles" zum Inbegriff einer vermeintlichen französischen Unterdrückungspolitik sowie zum Symbol für die Schwäche der neuen demokratischen Machthaber und ihren angeblichen Verrat an den "im Felde unbesiegten" Soldaten. In diesem Sinne diente der Rekurs auf den Erinnerungsort "Versailles" als Vorwand für außenpolitischen Revisionismus und innenpolitische Agitation gegen die politischen Führer der Weimarer Republik.

Die divergierenden Deutungen des Erinnerungsortes "Versailles" bestanden auch nach dem Zweiten Weltkrieg fort. In Frankreich blieb "Versailles" ein Symbol nationaler Einheit und Stärke, wurde nun allerdings - unter Ausblendung der durch Besatzung, Vichy-Regime und Résistance

\footnotetext{
${ }^{44}$ Zum Erinnerungsort "Versailles" siehe Hélène HimELFARB, "Versailles, fonctions et légendes", in NORA (Hrsg.), Les lieux de mémoire, Bd. II: La Nation, Teilbd. 2, Paris, Gallimard, 1986, S. 235-292; Jean-Claude AllAin, "Das Schloß von Versailles", in MöLLER / MORIZET (Hrsg.), Franzosen und Deutsche, S. 59-77; Hagen SCHUlze, "Versailles", in ders., FRANÇOIS (Hrsg.), Deutsche Erinnerungsorte, Bd. I, S. 407-421.
} 
entstandenen Risse in der nationalen Erinnerungskonstruktionen - eher wieder mit der vorrevolutionären und revolutionären Tradition in Verbindung gesetzt. In der Bundesrepublik wurde "Versailles", das die unbequeme Erinnerung an die Auswüchse des preußischen Militarismus mit der an die Kriegsniederlage und die Fehlentwicklungen der Zwischenkriegszeit verband, bestenfalls aus den vorherrschenden Erinnerungsdiskursen verbannt ${ }^{45}$. Wie tief verankert die gegensätzlichen erinnerungspolitischen Deutungen des Kriegsendes und des Versailler Vertrages in der deutschen und französischen Gesellschaft bis heute sind, illustrieren die bezeichnenderweise erst in jüngster Vergangenheit unternommenen Versuche, "Versailles" in einen gemeinsamen deutsch-französischen Erinnerungsort umzudeuten. So zielte die gemeinsame Sitzung des Bundestages und der Assemblée Nationale am 22. Januar 2003 im Schloss von Versailles darauf ab, den Mythos der deutsch-französischen "Aussöhnung" auf diesen im deutsch-französischen Zusammenhang bisher negativ gedeuteten Erinnerungsort zu übertragen und ihm dadurch eine neue Symbolik zu verleihen ${ }^{46}$. Der erwünschte Effekt blieb jedoch aus. Zwar gehören die Erinnerungskriege um "Versailles" heute sicher der Vergangenheit an. Zum Symbol der deutsch-französischen "Freundschaft" ist es freilich bis heute nicht geworden. Das Beispiel "Versailles" verweist daher - wie auch der ungeschickte und letztlich gescheiterte Versuch Nicolas Sarkozys, den 11. November und damit den Tag des Waffenstillstands 1918 zum "Tag der deutsch-französischen Aussöhnung" zu machen - auf die fortbestehenden Gegensätze zwischen den deutschen und französischen Erinnerungskulturen und die zentrale Bedeutung, die dem Ersten Weltkrieg nach wie vor im Aushandlungsprozess zwischen nationalem Gedenken und europäischer Geschichtspolitik zufällt.

\footnotetext{
45 Vgl. symptomatisch dazu einen zeitgenössischen Bericht über den Besuch von Altkanzler Adenauer 1966 im Versailler Schloss: Dieter WILD, "Nach Königskindern stand ihm nicht der Sinn", in Der Spiegel, 14.3.1966.

46 Vgl. Corine DeFRANCE, "Construction et déconstruction du mythe de la réconciliation franco-allemande", in Ulrich PFEIL (Hrsg.), Mythes et tabous des relations franco-allemandes au XX $X^{e}$ siècle - Mythen und Tabus der deutsch-französischen Beziehungen im 20. Jahrhundert, Bern, Lang, 2012, S. 69-85.
} 